\title{
Quarkonia as a multi-purpose tool
}

R. Vogt

January 12, 2011

Quarkonium 2010: Three Days of Quarkonium Production in $\mathrm{pp}$ and $\mathrm{pA}$ Collisions

Palaiseau, France

July 29, 2010 through July 31,2010 
This document was prepared as an account of work sponsored by an agency of the United States government. Neither the United States government nor Lawrence Livermore National Security, LLC, nor any of their employees makes any warranty, expressed or implied, or assumes any legal liability or responsibility for the accuracy, completeness, or usefulness of any information, apparatus, product, or process disclosed, or represents that its use would not infringe privately owned rights. Reference herein to any specific commercial product, process, or service by trade name, trademark, manufacturer, or otherwise does not necessarily constitute or imply its endorsement, recommendation, or favoring by the United States government or Lawrence Livermore National Security, LLC. The views and opinions of authors expressed herein do not necessarily state or reflect those of the United States government or Lawrence Livermore National Security, LLC, and shall not be used for advertising or product endorsement purposes. 


\title{
Quarkonia as a multi-purpose tool
}

\author{
R. $\operatorname{Vog} t^{\mathrm{a}, \mathrm{b}}$ \\ ${ }^{a}$ Physics Divsion, Lawrence Livermore National Laboratory, Livermore, CA 94551 \\ ${ }^{b}$ Physics Department, University of California at Davis, Davis, CA 95616
}

\begin{abstract}
Quarkonia can be a very useful tool for understanding the medium in which they are produced and pass through. However, their usefulness as a tool depends on how well certain aspects of their behavior in cold matter are understood.

Keywords: heavy flavor, production
\end{abstract}

The usefulness of the $J / \psi$ as a tool to probe the media depends on our understanding of its key features in normal matter including the production mechanism, singlet vs. octet; the level of feed down from the excited states, the $\psi(2 S)$ and $\chi_{c}$; and the nuclear gluon distribution. We discuss briefly how these features affect observables and then touch on how quarkonium can be used as a probe of the medium.

Unfortunately, our knowledge of the quarkonium production mechanism is still incomplete. While the leading order color singlet model clearly underpredicted the $J / \psi$ and $\psi^{\prime}$ cross sections at the Tevatron, the nextto-leading and incomplete next-to-next-to-leading order color singlet calculations do a much better job of describing the high $p_{T}$ data. Nonrelativisitic QCD, an expansion in the velocity of the $Q \bar{Q}$ pair as well as in the strong coupling constant, including both color singlet and color octet matrix elements, also agrees with the high $p_{T}$ data. The octet matrix elements are tuned to the data and used to predict other energies. Neither approach can provide an adequate description of the observed quarkonium polarization. The color-evaporation model, while also rather successful at describing the energy and $p_{T}$ dependence of quarkonium hadroproduction, cannot predict the polarization without significant deconstruction to obtain separate calculations of the different polarization states. See the new Quarkonium Working Group report [1] for a thorough discussion of the current theoretical status of quarkonium production.

The need for a detailed understanding of quarkonium production in hot matter is rather more prosaic. The nuclear dependence of quarkonium production in hadron-

Email address: vogt@physics.ucdavis.edu (R. Vogt) nucleus collisions is not under complete theoretical control. These cold matter effects include absorption of the quarkonium state, whether or not it interacts with the medium as a fully formed bound state or as a precursor state, nuclear modifications of the parton densities, energy loss while passing through the medium, and perhaps also quarkonium production through intrinsic charm, predicted to have a different nuclear dependence than the normal 'extrinsic' mechanism [2]. If the quarkonium state is subject to both hot and cold matter effects in nucleus-nucleus collisions, then it is necessary to have a handle on the effects of the absorption of the quarkonium state with bound nucleons.

Color singlets are expected to be absorbed with different cross sections than color octets [3]. Fast color octets have been expected to be absorbed with the same precursor cross section, independent of $x_{F}$ and the final quarkonium bound state. Thus the $J / \psi, \psi^{\prime}$ and $\chi_{c}$ would have the same absorption cross section. This is typically the absorption pattern assumed for predictions with the color evaporation model [4]. Color singlet absorption has generally been treated so that fast color singlet states passing through the nucleus are only absorbed if they hadronize while still in the medium. However, when the states can hadronize within the nucleus, they are expected to be absorbed with cross sections proportional to the square of their radii, obtaining their final state size at their formation times. Thus the $\chi_{c}$ and $\psi^{\prime}$, with longer formation times and larger radii than the $J / \psi$, should be absorbed more strongly when the absorption turns on. Inclusive $J / \psi$ absorption is an admixture of the direct contribution with the feed down from the $\chi_{c}$ and $\psi^{\prime}$. The boost of the formation time from the rest frame of the $J / \psi$ would suggest that, unless the formation times are 
very short, at forward $x_{F}$, the quarkonium states are still small color singlets when the emerge from the medium, thus suffering absorption only at relatively small or negative values of $x_{F}$ [3], meaning that differences in relative absorption might be hard to observe if this is the case. In NRQCD, the $J / \psi$ and $\psi^{\prime}$ are predominantly produced as color octets while the $\chi_{c}$ is almost entirely color singlet. Thus there should be an observable difference in absorption in this scenario.

Currently, the $\chi_{c} A$ dependence remains unmeasured. We know, however, that there exist measurements of $\psi^{\prime}$ relative to $J / \psi$ production in $p A$ interactions which show that, at least close to $x_{F} \sim 0$, the $\psi^{\prime}$ and $J / \psi$ effective absorption cross sections are significantly different $[5,6]$. Empirically, the difference also seems to decrease as a function of center of mass energy. Until the differences in production and absorption are fully under control, it is difficult to use quarkonium as a tool for extracting other properties of cold nuclear matter unless we can find an energy regime where absorption is negligible.

A recent study of the effective $J / \psi$ absorption cross section, $\sigma_{\text {abs }}^{J / \psi}$, found that $\sigma_{\text {abs }}^{J / \psi}$ seems to decreases with incident energy [7]. The value of the absorption cross section extrapolated back to incident energies of 158 $\mathrm{GeV}$, the same as the SPS nucleus-nucleus collisions, is significantly larger than that extracted at 400 and 450 $\mathrm{GeV}$, confirmed by recent NA60 data [8]. When modifications of the parton densites are included, via several parameterizations, the effective absorption cross section is increased at fixed-target energies since the parton momentum fractions probed are around $\langle x\rangle \sim 0.1$, in the antishadowing regime[7, 9]. At RHIC collider energies, the effective absorption cross section should be smaller but not negligible. If $\sigma_{\text {abs }}^{J / \psi}$ approaches zero asymptotically, absorption may well be negligible at the LHC [10], as we should find out in the next few years since confirmation must necessarily wait for an LHC $p A$ run.

Until such time that absorption can be proved negligible in the energy regime of interest, using the $J / \psi$ to constrain the gluon density, while appealing, is likely not feasible. However, if absorption is negligible, then the ratio of the $p A$ (or $\mathrm{d} A$ ) to $p p$ cross sections, if taken at the same energy, provides a measure of the modifications of the low $x$ gluon density in the nucleus at higher scales than previously available [10],

$$
\frac{\sigma_{p A}\left(\sqrt{s_{N N}}\right)}{\sigma_{p p}\left(\sqrt{s_{N N}}\right)} \propto \frac{1}{A} \frac{f_{g}^{A}\left(x, \mu^{2}\right)}{f_{g}^{p}\left(x, \mu^{2}\right)} .
$$

If the $p A$ center of mass energy is different from the $p p$ energy, as may be expected for LHC measurements in

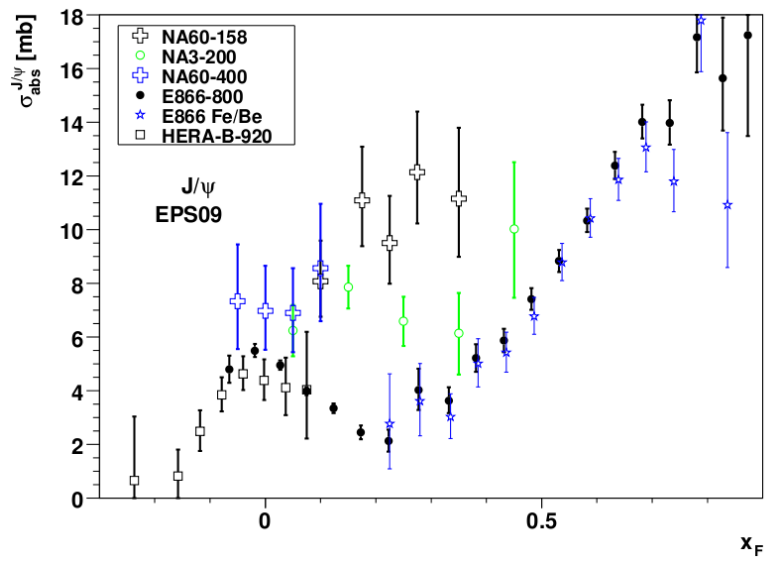

Figure 1: The $x_{F}$ dependence of $\sigma_{\mathrm{abs}}^{J / \psi}$ for incident fixed-target energies from 158 [8] 200 [12], 400 [5], 450 [13], 800 [6] and 920 [14] GeV obtained using the EPS09 shadowing parameterization [15].

the equal-speed frame [10], then the $x$ values are not the same and the extraction of the gluon modifications is less straightforward,

$$
\frac{\sigma_{p A}\left(\sqrt{s_{N N}}\right)}{\sigma_{p p}(\sqrt{s})} \propto \frac{1}{A} \frac{f_{g}^{A}\left(x^{\prime}, \mu^{2}\right)}{f_{g}^{p}\left(x, \mu^{2}\right)} .
$$

We have not mentioned initial state energy loss. The level of initial state energy loss predicted from extraction of quark energy loss in the Drell-Yan process, may be expected to be small [11]. Possible final-state loss on the formed $J / \psi$ has not yet been considered. We note that fractional energy loss effects (e.g. $\left.x_{1}^{\prime}=x_{1}-\Delta x_{1}\right)$ are only sizable when $x_{1}$ is large. Thus energy loss effects turn on at values of $x_{1} \sim 0.1$, close to $x_{F} \sim 0$ at $158 \mathrm{GeV}$ but moving to higher, more forward values of $x_{F}$ with increasing energy. At RHIC, this region may be pushed to a rapidity range near the edge of the observable region. Figure 1 shows the effective absorption cross section as a function of $x_{F}$ for incident proton energies from $158 \mathrm{GeV}$ to $920 \mathrm{GeV}$. The only additional cold matter effect included is shadowing. Thus the increase in the absorption cross section with $x_{F}$ may be due to the turn on of final-state energy loss, The turn on comes at higher $x_{F}$ since non-negligible values of $x_{1}$ move to higher $x_{F}$ with increasing incident energy.

Once cold matter effects are under control, $J / \psi$ production in hot matter can be used to probe the medium in numerous ways. If $J / \psi$ production is strongly suppressed in the medium, the only observed $J / \psi$ may be produced at late times, either by $B$ decays or by coalescence of $c \bar{c}$ pairs. In the latter case, the $c \bar{c}$ pairs that produce the $J / \psi$ may be either correlated (same initial 
pair) or uncorrelated (two different initial pairs). These secondary $J / \psi$ are expected to have narrower rapidity distributions and lower average $p_{T}$ [16]. There are hints that such production is part of the observed $J / \psi$ 's at RHIC [17].

In hot matter, the quarkonium potential has been modeled both in terms of the color-singlet free energy $F$ and the internal energy $U=F-T \partial F / \partial T$. Recently Riek and Rapp have looked into the consequences of both cases on the charm quark equilibration (relaxation) times [18]. The free energy results in rather weak binding where the quarkonium $S$ wave dissociation temperature is on the order of $1.3 T_{c}$ while choosing $U$ results in stronger, tighter quarkonium binding with $S$ wave dissociation only at $T \sim 2 T_{c}$. The strong-binding scenario results in a longer $c$-quark relaxation time than the weak-binding case, $\tau=6$ and $3 \mathrm{fm} / c$ respectively. The coalesence contribution can be tuned in the two cases to satisfactorily explain the centrality dependence of $J / \psi$ suppression at the SPS and RHIC with a larger coalescence contribution necessary in the weak-binding scenario. It might be possible to distinguish between strong and weak-binding scenarios by an examination of the $p_{T}$ dependence of $J / \psi$ suppression [19]. Thus the $J / \psi$ could be used to measure the coupling strength of the medium as well as serve as an equilibration chronometer.

The amount of $J / \psi$ elliptic flow in hot matter could be used to measure the relative thermalization of the medium. Initially produced $J / \psi$ 's are not expected to flow while charm quarks have been shown to flow (at least through measurements of non-photonic electrons) [17]. The $p_{T}$ dependence of the suppression factor $R_{A A}$ as a function of $p_{T}$ also measures the system size and coupling strength of the system.

For quarkonium to be an effective tool, its production and suppression needs to be studied over a range of incident energies and system sizes. Studying a range of energies would vary the initial quarkonium production rate while changing the $x$ region probed; verify the energy dependence of the absorption cross section; and adjust the temperature and density of the nucleus-nucleus collision system for fixed nuclear mass. Using a variety of nuclear targets/beams for fixed-target/collider systems alters the system size and path length traversed by the quarkonium state; adjusts the temperature and density of the system at a fixed energy; and allows a study of volume relative to area effects.

Finally, quarkonium studies should not neglect bottomonium production and suppression effects. Since the $\Upsilon(1 S)$ is smaller than the $J / \psi$, its absorption cross section should be smaller still. The larger $\Upsilon$ mass probes shadowing and energy loss effects at a larger scale thus larger $x$ values than the $J / \psi$. In terms of nucleusnucleus collisions, the $\Upsilon$ is expected to dissociate at a higher temperature than all the other quarkonium states, thus proving to be a more effective thermometer of the system. In addition, the smaller $b \bar{b}$ rate results in a lower probability for production by coalescence [17].

\section{Acknowledgments}

The work of R.V. was performed under the auspices of the U.S. Department of Energy by Lawrence Livermore National Laboratory under Contract DE-AC5207NA27344 and was also supported in part by the National Science Foundation Grant NSF PHY-0555660.

\section{References}

[1] N. Brambilla et al., arXiv:1010.5827 [hep-ph].

[2] R. Vogt, S. J. Brodsky and P. Hoyer, Nucl. Phys. B 360 (1991) 67.

[3] R. Vogt, Nucl. Phys. A 700 (2002) 539 [arXiv:hep-ph/0107045].

[4] R. Vogt, Phys. Rev. C 71 (2005) 054902 [arXiv:hep$\mathrm{ph} / 0411378]$.

[5] B. Alessandro et al. [NA50 Collaboration], Eur. Phys. J. C 48 (2006) 329 [arXiv:nucl-ex/0612012].

[6] M. J. Leitch et al. [FNAL E866/NuSea collaboration], Phys. Rev. Lett. 84 (2000) 3256 [arXiv:nucl-ex/9909007].

[7] C. Lourenco, R. Vogt and H. K. Woehri, JHEP 0902 (2009) 014 [arXiv:0901.3054 [hep-ph]].

[8] E. Scomparin [NA60 Collaboration], Nucl. Phys. A 830 (2009) 239c [arXiv:0907.3682 [nucl-ex]].

[9] R. Arnaldi, P. Cortese and E. Scomparin, Phys. Rev. C 81 (2010) 014903 [arXiv:0909.2199 [hep-ph]].

[10] R. Vogt, Phys. Rev. C 81 (2010) 044903 [arXiv:1003.3497 [hep$\mathrm{ph}]$.

[11] H. K. Wörhi, these proceedings.

[12] J. Badier et al. [NA3 Collaboration], Z. Phys. C 20 (1983) 101.

[13] B. Alessandro et al. [NA50 Collaboration], Eur. Phys. J. C 33 (2004) 31.

[14] I. Abt et al. [HERA-B Collaboration], Eur. Phys. J. C 60 (2009) 525 [arXiv:0812.0734 [hep-ex]].

[15] K. J. Eskola, H. Paukkunen and C. A. Salgado, JHEP 0904 (2009) 065 [arXiv:0902.4154 [hep-ph]].

[16] R. L. Thews and M. L. Mangano, Phys. Rev. C 73, 014904 (2006) [arXiv:nucl-th/0505055].

[17] A. D. Frawley, T. Ullrich and R. Vogt, Phys. Rept. 462 (2008) 125 [arXiv:0806.1013 [nucl-ex]].

[18] F. Riek and R. Rapp, Phys. Rev. C 82 (2010) 035201 [arXiv:1005.0769 [hep-ph]].

[19] X. Zhao and R. Rapp, arXiv:1008.5328 [hep-ph]. 